\title{
In-situ repair/maintenance with a continuum robotic machine tool in confined space
}

\author{
Xin Dong a , David Palmer a, Dragos Axinte ${ }^{a}$, James Kell b \\ a. Rolls-Royce University Technology Centre in Manufacturing and On-wing Technology, Faculty of \\ Engineering, University of Nottingham, United Kingdom; \\ b. Repair Technology, Rolls-Royce plc, United Kingdom;
}

Corresponding author: Xin Dong (Xin.Dong@nottingham.ac.uk)

\begin{abstract}
In-situ repair/maintenance is critical for the key industry sectors (e.g. aerospace, nuclear), which require prompt interventions. The paper reports on a tendon driven continuum robotic machine tool with high manoeuvrability in navigating and machining within confined workspaces. The design reveals the main working principles of the robotic machine tool, followed by its calibration method and a new kinematics compensation approach to account for the deformation of the actuation cables that is that the key enabler for its use in machining tasks, which is based on the geometry of the actuation system. Finally, a set of experimental trials were conducted to show its ability to perform in-situ accurate machining operations in a mock-up aerospace scenario.
\end{abstract}

Key words: continuum robotic machine tool, mechanical design, kinematics, calibration, machine trajectory.

\section{Introduction}

In-situ maintenance works (e.g. inspection and repair) have significant impacts on the operation of high value-added products (e.g. aero-engines), since it can shorten both the time and cost of such interventions [1]. In many cases, the in-situ interventions are performed by bespoke tools in confined space [2]. Further, some versatile systems, e.g. industrial robots and portable machine tools, have also been utilised for repair applications $[3,4]$. However, due to the size of the systems, these portable machine tools are more suitable for the repairs in open space rather than the restricted ones.

Recently, the use of continuum robots has shown that they can be capable to provide some of the expected performances in difficult-to-access environments. For example, rigid backbone continuum robots employ one universal joint (2DOF) in each section, which can be utilised for the inspection in aircraft wings and nuclear facilities. However, due to the limited number of the Degree-of-freedom (DoF, including both active and passive) along the arm, it is still difficult to access into crammed space. In comparison, more DoFs are integrated in the continuum robots utilised in minimally invasive surgeries for monitoring and removing soft tissues, leading to better accessibility but less stiffness and loading capability [5, 6]. However, machining operations, which require long arm, appropriate stiffness and tool path planning, have never been reported with this type of complex manipulators. Further, there are different approaches of actuation design, according to the number of actuations in single section. Most of the designs employ three motors for manipulating single section [7], while the others hire two motors with spool mechanisms for pulling two pairs of cables in single section [8, 9].

Significant efforts in the area have been taken on the kinematics of the continuum robots. Most of the models take an assumption of constant curvature of the continuum robot backbone. For example, virtual work approach was utilised for solving the kinematics of a minimally invasive surgery continuum robot, which is composed of three motors in each section [10, 11]. In this model, all the cables are independently driven by motors and their tension can be kept well. Furthermore, a geometrical method was utilised to derive the kinematics for the continuum robots with two actuators in each section [12]. However, it seems that the cable slack caused by the spool mechanism in the two-actuator structure has not been considered in the. Hence, the 
cable slack needs to be considered in the kinematics model, in order to minimise the positional error of the end effector.

This paper presents the development and machining trials of a flexible slender continuum robotic machine tool, which has a set of key abilities: (i) navigation into the intervention workspace; (ii) machining operation for repair; (iii) inspection operation, in cluttered environments. Further, the kinematics model with cable slack compensation is presented for improve the positional accuracy of the system and an algorithm for generating tool path is proposed for supporting the in-situ repair operation in confined space. Finally, a demonstration was conducted to evaluate the machining capability of the continuum robotic machine tool.

\section{A continuum robot as machine tool}

In the following, the specifications of the continuum robotic machine tool system with the kinematic model and a backlash compensation approach are presented.

\subsection{Novel design of the continuum robot system}

Unlike the conventional serial machine tools, the continuum robot arm $(300 \mathrm{~mm}$ long and $15 \mathrm{~mm}$ in diameter) is actuated remotely by the actuators located at the base of the system via cables, which renders its size significantly smaller [8].

The continuum arm is structured with three 2-DoF sections (Fig. 1a and b) which is composed of multiple groups of compliant joints (made of super-elastic Nitinol) connected disks, which makes it easier for accessing into confined space. In the robotic arm, there is an $8 \mathrm{~mm}$ through hole, which can pass different tools to the end of the arm (e.g. camera and grinding tools).

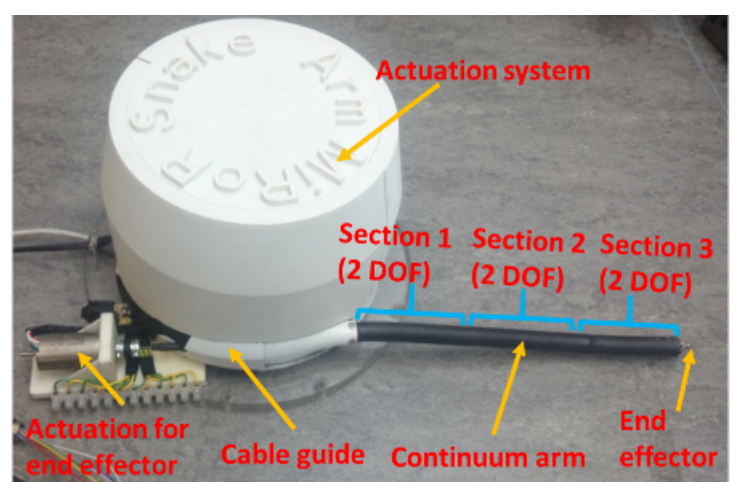

(a)

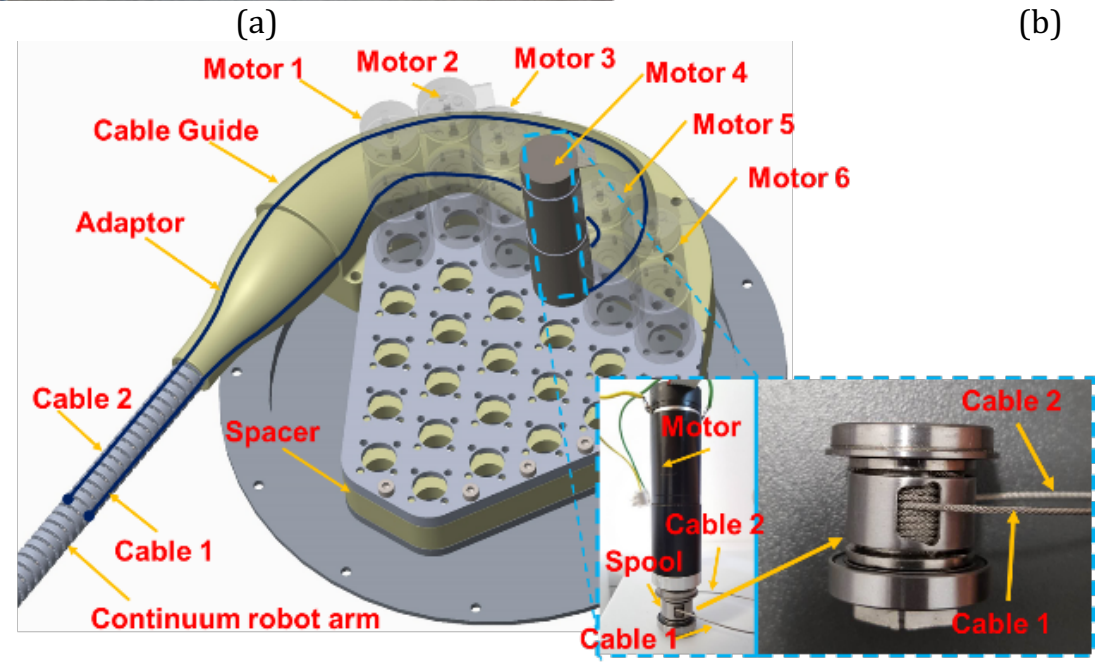

(c)

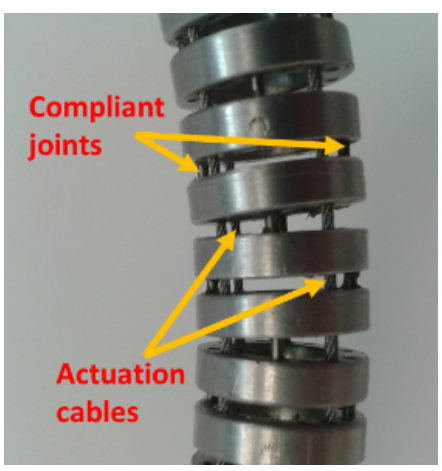

(b)

Fig. 1. 6 Degree of freedom (DOF) continuum robotic machine tool (a) with its structure (b) and actuation system - only one motor shown (c) 
Every section (100mm in length) is actuated by two motors via two pairs of antagonistic steel cables. Specifically, each pair of the cables is attached to a motor by its spool, then sequentially go through the cable channels of a spacer, cable guide and an adaptor to the continuum robot arm, finally attached to the last disk of each section (Fig. 1c). In each section, the space and weight of one motor is saved by the spool mechanism, rendering the actuation system lighter and smaller, compared with the other designs (most of the continuum robot arms employ three motors for each section). However, this novel design solution also brings a kinematics challenge for the manipulator control, since the tension of each individual cable in the spool varies during the rotation (it is discussed in the following section). Hence, although the kinematics of the continuum arm was developed, a compensation approach for it still needs to be developed for minimizing the error caused by the cable slack, which is essential for in-situ interventions, machining repair.

\subsection{Modelling of the continuum robot arm}

Unlike conventional robots, for controlling a 6-DoF continuum arm, there are two levels of kinematics need to be studied, i.e. kinematics in actuation and configuration spaces. In actuation space, the kinematics studies the mapping between the actuation (i.e. motor) displacement and the configuration of a section (i.e. bending $(\varnothing)$ and direction $(\varphi)$ angles, Fig. 2), while in configuration space, it studies the relation between the arm tip position and the configuration of each section. Hence, driving a continuum arm to a given position needs the following steps:

1. Calculate the pose of each section according to the given position (i.e. configuration space kinematics);

2. Calculate the displacement of each motor based on the poses from step 1 (i.e. actuation space kinematics).

Since the cable slack occurs in the actuation level, kinematics in actuation space is investigated by considering the cable slack error and the geometry of the system. As shown in Fig. 2, the structure of a segment within a section of the continuum arm, which is the basic element in a section containing three disks and two perpendicularly placed pairs of compliant joints, is presented with its kinematics model.

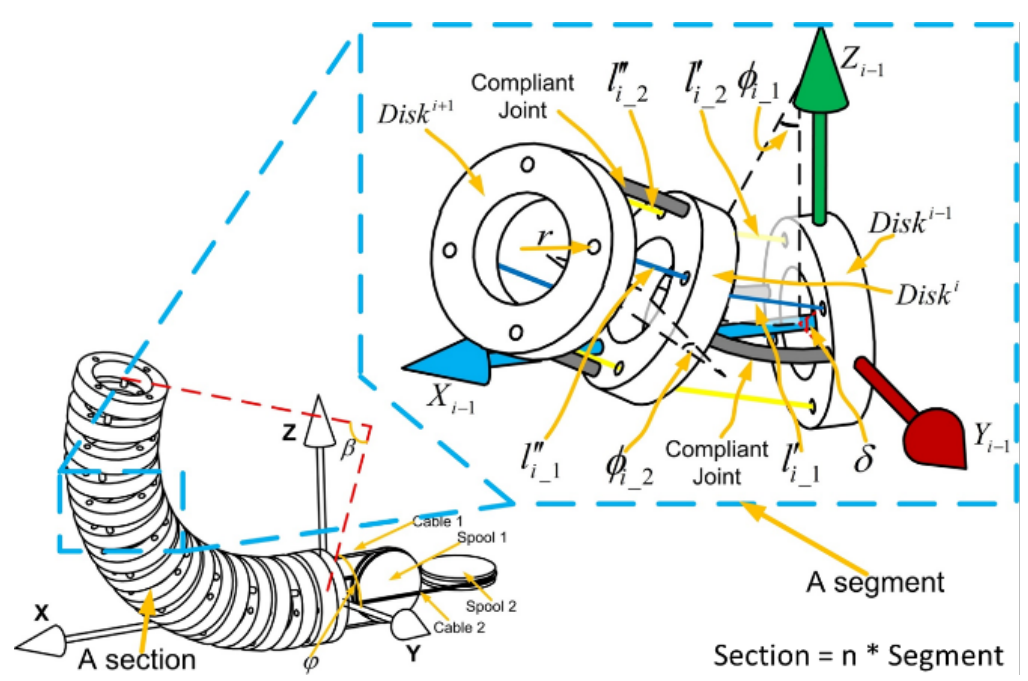

Fig. 2. Kinematics model of the continuum arm

Firstly, assuming the cable can maintain the tension at arbitrary bending configuration (without any backlash) and taking into consideration of the geometrical notations in Fig. 2, the inverse kinematics of the continuum robot arm is presented in Eq. 1 to compute cable length $\left(l_{i_{-} 1}, l_{i_{-}}\right)$ based on the section configuration $(\varnothing, \varphi)$ : 


$$
\left\{\begin{array}{l}
l_{i_{-} 1}=2 n\left(\frac{l_{c}}{\phi_{i-1}}-r \sin \delta\right) \tan \left(\frac{\phi_{i-1}}{2}\right)+2 n\left(\frac{l_{c}}{\emptyset_{i-2}}-r \cos \delta\right) \tan \left(\frac{\phi_{i-2}}{2}\right) \\
l_{i_{-} 2}=2 n\left(\frac{l_{c}}{\emptyset_{i-1}}-r \cos \delta\right) \tan \left(\frac{\phi_{i-1}}{2}\right)+2 n\left(\frac{l_{c}}{\emptyset_{i_{-} 2}}-r \sin \delta\right) \tan \left(\frac{\phi_{i-2}}{2}\right)
\end{array}(i=1,2, \ldots n)\right.
$$

Where, $\mathrm{n}$ is the number of segments in a section; $s \delta=\sin (\delta) ; c \delta=\cos (\delta) ; l_{c^{-}}$- compliant joint length; $\emptyset_{i_{1} 1}$ and $\emptyset_{i \_2}$ are the bending angles between $D i s k^{i-1}$ and $D_{i s k^{i}}$, between $D_{i s k^{i}}$ and $D i s k^{i+1}$, respectively; $\delta$ - the offset angle of cable $1\left(l_{i_{1}}^{\prime}\right)$ hole from the axis $Y_{i-1}$;

Then, the cable slack is considered in the kinematics modelling. The cables are helically coiled on the spool (Fig. 3a), thus, one cable contracts and the other one extends, so that a section can be bent. However, the cable lengths of contracting and extending are not precisely equal (discussed in the following of the chapter), causing one cable is well-tensioned (contracting and bending the arm) and the other one is slack (extending), which leads to a backlash phenomenon on controlling the robot arm.

In order to estimate the backlash, the kinematics of the spool mechanism is studied, where the cable is coiled in a helix spiral pattern (Fig. 3b), which is defined in Eq.2 as:

$$
\left\{\begin{array}{c}
x=d \cdot \sin \theta \\
y=d \cdot \cos \theta \\
z=a \cdot \theta /(2 \pi)
\end{array}\right.
$$

Where: $\mathrm{d}$ - distance between spool centre and cable centre line, $a$ - cable diameter; $\theta$ - spool rotation angle from the initial position;

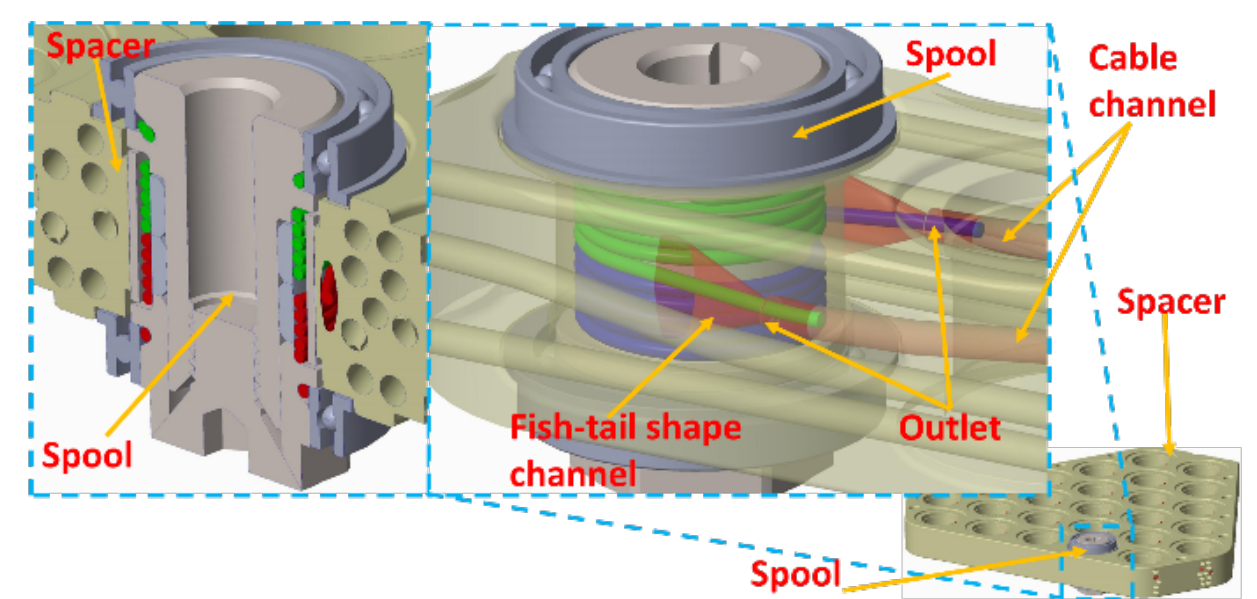

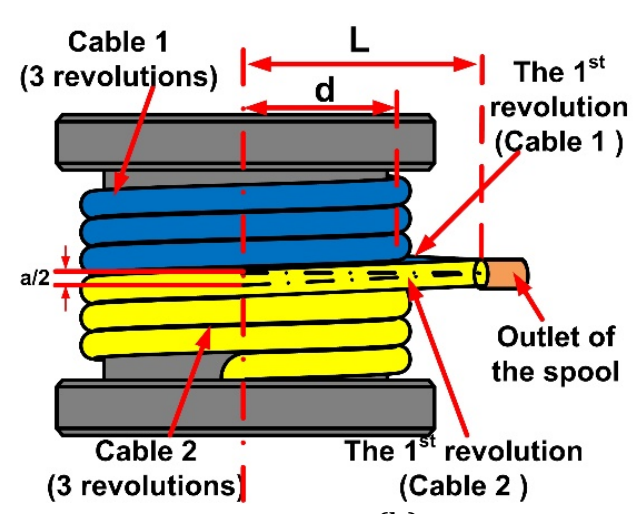

(b) (a)

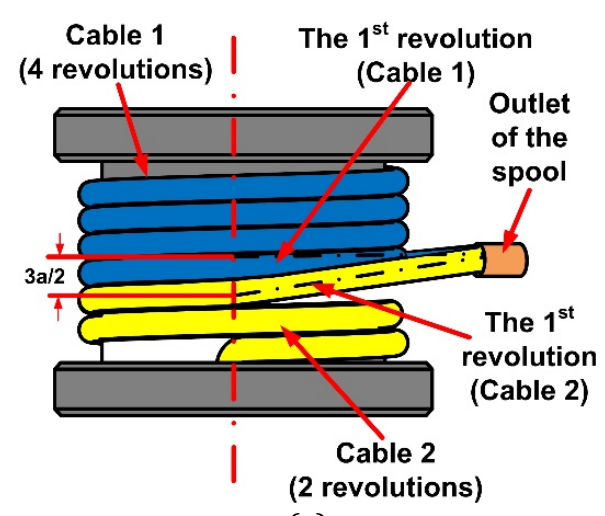

(c) 
Fig. 3. Structure of the special spool, with spacer with its channels, that allow the use of only two motors for actuation per each 2-DoF sections (a); kinematics model of the spool mechanism: the cable layout at the initial position - 3 revolutions coiled on the spool for each cable (b); the cable layout for one revolution spool rotation -4 revolutions coiled on the spool from cable 1 (blue) and 2 revolutions from cable 2(yellow) (c);

However, the 1st revolutions of cable 1 and 2 (Fig. 3b and c) are always in different horizontal planes, rendering the contacting cable length of the spool is different from the extending cable length, which causes the backlash problem. Specifically, based on the geometry parameters of the spool, the displacement of cable 1 (contracting) against the rotational angle of the spool $(\theta)$ can be expressed as:

$$
l_{1}=\frac{\theta}{2 \pi} l_{H}+\sqrt{\left(\frac{\theta}{2 \pi} a+\frac{a}{2}\right)^{2}+l^{2}}-\sqrt{\left(\frac{a}{2}\right)^{2}+l^{2}}
$$

Where the length of one revolution of helix wire $l_{H}=\sqrt{(2 \pi r)^{2}+a^{2}} ; \sqrt{(\theta \cdot a / 2 \pi+a / 2)^{2}+l^{2}}-\sqrt{(a / 2)^{2}+l^{2}}$ is the resultant cable length change from the spool centre to the outlet. Similarly, the displacement of cable 2 (extending) can be expressed as:

$$
l_{2}=\frac{\theta}{2 \pi} l_{H}+\sqrt{\left(\frac{\theta}{2 \pi} a-\frac{a}{2}\right)^{2}+l^{2}}-\sqrt{\left(\frac{a}{2}\right)^{2}+l^{2}}
$$

The difference between the contracting and extending cable lengths in the pair can be calculated by:

$$
\Delta l=\sqrt{\left(\frac{\theta}{2 \pi} a+\frac{a}{2}\right)^{2}+l^{2}}-\sqrt{\left(\frac{\theta}{2 \pi} a-\frac{a}{2}\right)^{2}+l^{2}}
$$

From Eq.5, it can be found that the difference between the contracting and extending cable length and the slack is from the angle between the spool cores to the cable outlet. Based on the finding from Eq.5, it has been corrected in the new control program of the continuum robot arm to enable precise positioning of the end effector (micro-spindle) suitable for in-situ machining.

\section{Calibration and machining trajectory}

In order to perform in-situ repair operations, two preparatory steps (i.e. calibration and tool path algorithm), that consider the cable slack compensation method, need to be conducted before the repair operation, which are studies in the following part.

\subsection{Calibration}

Firstly, a novel calibration approach is presented, which provides the relative position of the workpiece in the coordinate of the continuum robot arm. Based on the defect position identified from a camera embedded in the robot tip, the calibration process was conducted by the following steps:

1. Move the system linearly towards to the engine by a linear stage, meanwhile navigate the arm to target area (Fig. 4a).

2. Move the end effector of the continuum robot arm (i.e. tool) till it touches the edge of the target at $\mathrm{P}_{1}\left(\mathrm{x}_{1}, \mathrm{y}_{1}, \mathrm{z}_{1}\right)$ (Fig. $4 \mathrm{~b}$ ), which is the start of a scallop shape (that is to be ground) and save the parameters of each section $\left(\beta_{i}^{P 1}, \varphi_{i}^{P 1}\right)(i=1,2,3)$ and the tip position of each section $P_{i_{-1}}(i$ $=1,2,3$ )

3. Move the end effector to touch the edge of the target at $P_{2}\left(x_{2}, y_{2}, z_{2}\right)$ (Fig. 4c), which is the end of the scallop shape grinding and save the parameters of each section $\left(\beta_{i}^{P 2}, \varphi_{i}^{P 2}\right)(i=1,2,3)$ and the tip position of each section $P_{i_{-} 2}(i=1,2,3)$; 


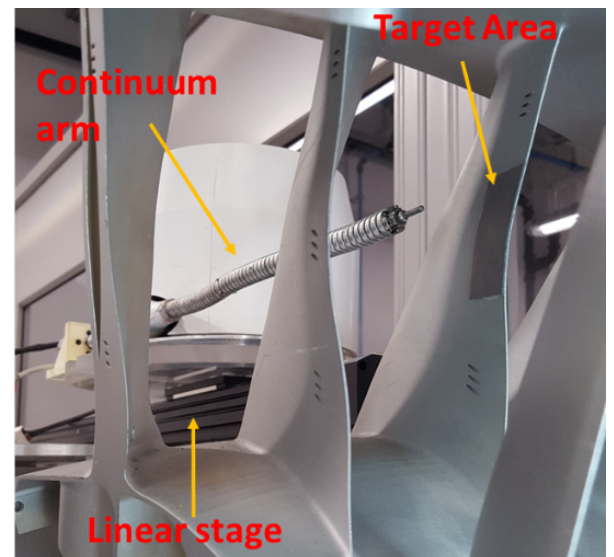

(a)

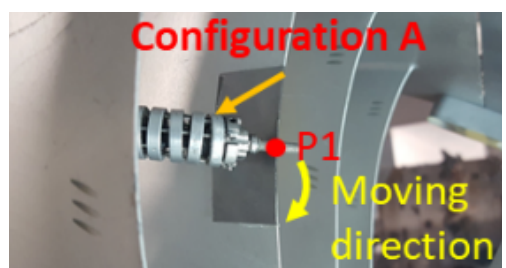

(b)

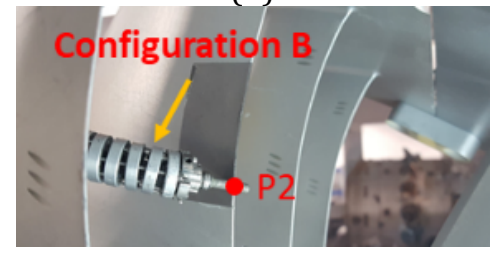

(c)

Fig. 4. The system set up and the calibration process

Further, when the tool touches the workpiece, the angle between the workpiece and the tool $(\alpha)$ needs to be maintained in a range between $90^{\circ}$ and $50^{\circ}($

Fig. 5). As the tool path is generated on the normal plane of the tool, the depth of the cut in the workpiece can be expressed as $D_{2}=D_{1} / \sin (\alpha)$, where $D_{1}$ is the tool path depth (when $\alpha_{\min }$ is $50^{\circ}, D_{2}=1.3 \times D_{1}$; when $\alpha_{\max }=90^{\circ}, D_{2}=D_{1}$ ) in the normal plane of the tool. As $D_{1}$ is significantly less than $1 \mathrm{~mm}$, the max difference between $D_{1}$ and $D_{2}$ can be maintained under $0.32 \mathrm{~mm}\left(0.3 \mathrm{D}_{1}\right)$.

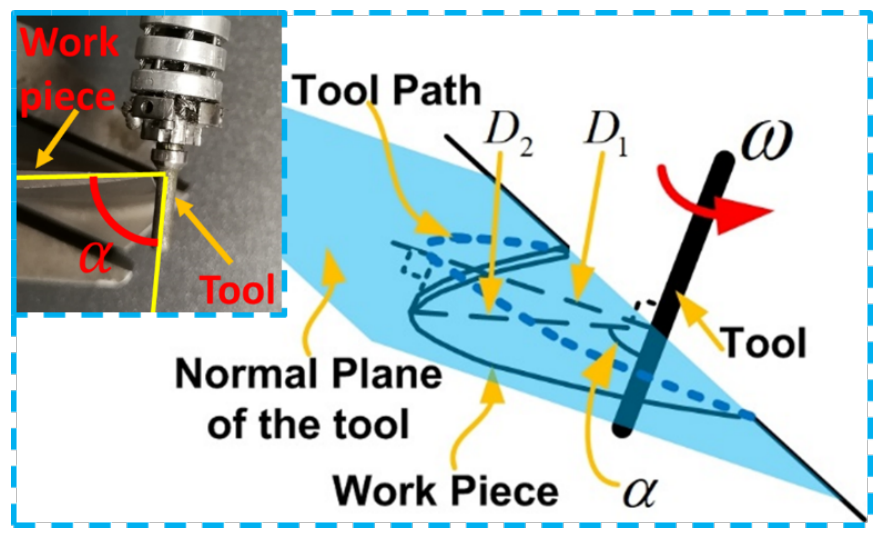

Fig. 5. The machining angle between the tool and the workpiece

\subsection{Tool Path Algorithm}

One of the most important applications for the continuum robotic machine tool is in-situ repair of dents in the rotating parts of aero-engines. Hence, in this paper, an example of tool path algorithm for removing a scallop shape material from the workpiece (the length is significantly longer than its depth) is presented. For this, a series of tool path needs to be generated to remove the material layer by layer. After initializing points $P_{1}$ and $P_{2}$, the tool of the continuum robot arm can interpolate between these points and perform the scallop in the desired direction. 


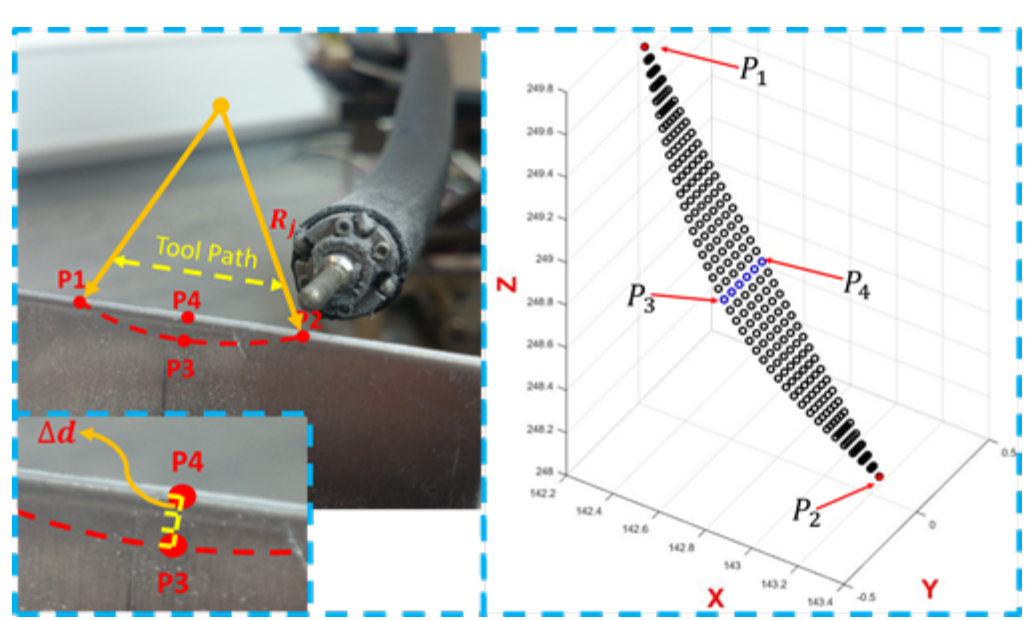

Fig. 6. Schematic diagram of the scallop cut (the right figure is an example generated by the tool path algorithm in MATLAB)

As show in Fig. 6, a tool path for a scallop shape grinding can be determined as a part of a circle by the following equation, which is in the plane normal to the tool of the manipulator (assuming the required depth/length ratio of scallop cut is $1: \mathrm{w}$ (generally between 1:4 and 1: 10), the length of the scallop $P_{1} P_{2}$ is w times of the depth $P_{3} P_{4}$.):

$$
R_{j}^{2}=\left(R_{j}-j \cdot \Delta d\right)^{2}+\left(\frac{w}{2} P_{3} P_{4}\right)^{2}(\mathrm{j}=1,2, \ldots \mathrm{n})
$$

Where: $\Delta d$ - step between the adjacent layers and $R_{j}$ - radius of the path. There, the radius of the tool path for layer $\mathrm{j}$, can be obtained:

$$
R_{j}=\frac{\left(3 P_{3} P_{4}\right)^{2}+(j \cdot \Delta d)^{2}}{2 j \cdot \Delta d}(\mathrm{j}=1,2, \ldots \mathrm{n})
$$

Hence, a tool path can be defined, which is circular arc passing points $P_{1}$ and $P_{2}$ with a diameter $R_{j}$.

Further, in order to drive the tool along planned path, an array of the continuum arm configurations is required, which can be generated by the following steps:

1. Split evenly distributed points (quantity in one layer: $\mathrm{m}$ ) along the path between the two calibration points $\left(P_{3_{-} k}, \mathrm{k}=1\right.$ to $\left.\mathrm{m}, \mathrm{m} \geq 2 ; P_{1}=P_{3_{-} 1} ; P_{2}=P_{3_{-}}\right)$.

2. For each point of the tool path, the parameters of each section of the manipulator $\left(\beta_{i}^{P_{k}}, \varphi_{i}^{P_{k}}\right.$, $\mathrm{k}=1$ to $\mathrm{m}, \mathrm{m} \geq 2 ; i=1$ to 3 ) are calculated by inverse kinematics in configuration space.

Specifically, inverse kinematics in the configuration space studies the poses (position, orientation) of all the sections for a given tool centre point location. Unlike the previous numerical methods for solving the inverse kinematics of 6-DOF continuum robot [13], a new approach was developed in this paper, by calculating the inverse kinematics with a closed-form equation, which is more computationally effective. 


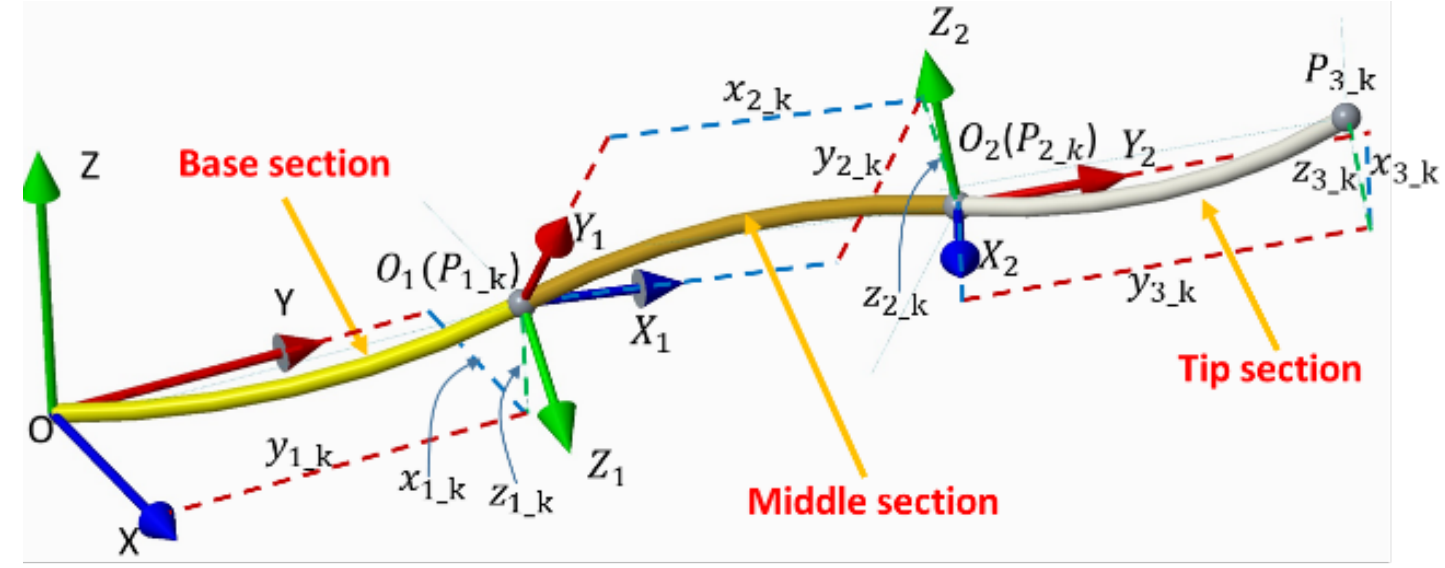

Fig. 7. Inverse kinematics of 6 DOF robot arm in configuration space

The detailed derivation of the inverse kinematics in configuration space is presented (Fig. 7). For the base section, the positons of the starting point $\left(P_{1_{1} 1}\right)$ and the end point $\left(P_{1_{-} \mathrm{m}}\right)$ are obtained during the calibration, which are the start and end points of the section tip trajectory corresponding to the tool path. Since the distance between $P_{1_{-} 1}$ and $P_{1_{-} m}$ is short, the tip position trajectory between these two points is assumed as linear, thus, the points between these two points can be planned by Eq. 8 .

$$
\overrightarrow{O P_{1 \_k}}=k \cdot \frac{\overrightarrow{O P_{1-1}}+\overrightarrow{O P_{1 \_}}}{m-1}(\mathrm{k}=1,2, \ldots \mathrm{m} ; \mathrm{m} \geq 2)
$$

Where, $\mathrm{m}$ is the total number of the points along the tool path and $\overrightarrow{O P_{1 \_k}}\left(x_{1 \_\mathrm{k}}, y_{1 \_\mathrm{k}}, z_{1 \_\mathrm{k}}\right)$ is descried in coordinate OXYZ. Hence, the bending and direction angle of the section at $P_{1 \_\mathrm{k}}$ can be written in the following form:

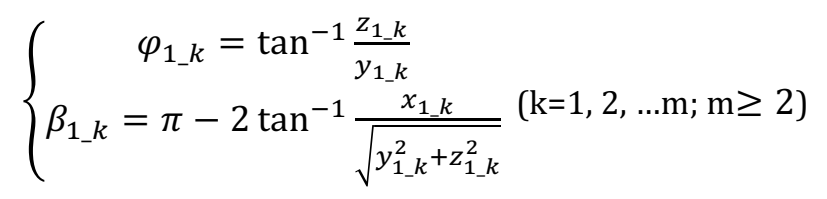

Thus, the orientation matrix of the base section $\left(T_{1 \_\mathrm{k}}\right)$ at point $P_{1 \_\mathrm{k}}$ can be calculated, which also defines the coordinate $O_{1} X_{1} Y_{1} Z_{1}\left(T_{1 \_k}, \overrightarrow{O P_{1 \_k}}\right)$. Similarly, based on the calibration, the tip point $P_{2 \_\mathrm{k}}$ of the middle section can be obtained, which is descried in the coordinate OXYZ. In order to calculate the bending and direction angle of the middle section, $P_{2 \_\mathrm{k}}\left(x_{2 \_}, y_{2 \_}, z_{2 \_} \mathrm{k}\right)$ needs to be described in the coordinate $O_{1} X_{1} Y_{1} Z_{1}$, as in Eq. 10:

$$
\overrightarrow{O_{1} P_{2 \_} k}=T_{1 \_k} \cdot \overrightarrow{P_{1 \_k} P_{2 \_} k}(\mathrm{k}=1,2, \ldots \mathrm{m})
$$

Where, $\overrightarrow{O_{1} P_{2 \_}}$is the tip position of the middle section described in coordinate $O_{1} X_{1} Y_{1} Z_{1}$ and $\overrightarrow{P_{1 \_k} P_{2 \_} k}$ is described in coordinate OXYZ. According to Eq. 9 and 10, the bending and direction angle of the middle section can be calculated. Similarly, the configuration of the tip section can be obtained. Therefore, the parameters of all the sections for tool path split point are calculated.

Finally, the whole procedure for the calibration and tool path generation is presented in Fig. 8. 


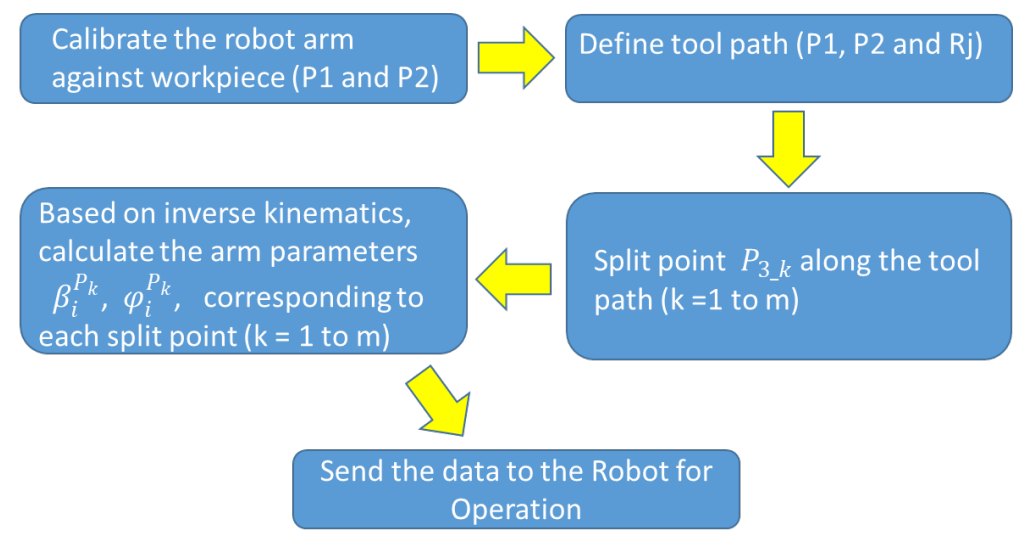

Fig. 8. Tool path generation process for the 6-DoF continuum robot considering the inverse kinematics

Overall, the proposed path generation method can be utilised for producing a particular scallop shape of the trajectories for removing the cracks on the compressors, which is a part of a circle. After the start and end points of the trajectory defined, the size of the trajectory can be controlled by applying different required depth/length ratios w. Although this method cannot be utilised for non-circular trajectories, it still can meet the requirements of most of the repair cases.

\section{Demonstration and result}

In order to validate the proposed methods and algorithms (i.e. the cable backlash compensation method, calibration approach and tool path generation algorithm), several machining trials were conducted in an aero-engine mock-up, which demonstrates the machining capability of the continuum robot arm in confined spaces (Fig. 9a). In these trials, scallop shape material removing (as an example, grinding depth $=1 / 6$ of length) were tested at the following different configurations.

1. Configuration $1-$ Base Section: $\beta_{1}=20^{\circ}, \varphi_{1}=-35^{\circ}$; Middle Section: $\beta_{2}=10^{\circ}, \varphi_{2}=0^{\circ}$; Tip Section: $\beta_{3}=60^{\circ}, \varphi_{3}=180^{\circ}$;

2. Configuration $2-$ Base Section: $\beta_{1}=46^{\circ}, \varphi_{1}=30^{\circ}$; Middle Section: $\beta_{2}=10^{\circ}, \varphi_{2}=0^{\circ}$; Tip Section: $\beta_{3}=56^{\circ}, \varphi_{3}=180^{\circ}$;

The tested parts of the components in the engine mock-up are made from Ni-based alloy, where the scallop cuts are max $3 \mathrm{~mm}$ in length. The cutting speed of the tool was $160 \mathrm{~m} / \mathrm{min}$, which is driven by a Maxon motor (EC-4pole $30 \varnothing 30 \mathrm{~mm}$, brushless, $100 \mathrm{Watt}$ ) via a torque cable (length: $400 \mathrm{~mm}$, diameter: $2.5 \mathrm{~mm}$, max torque: $2 \mathrm{Nm}$ ) at the linear speed of $0.5 \mathrm{~mm} / \mathrm{s}$.

According to the results measured from the machined feature on the components, it can be found that removing the material in the shape of a scallop (length is $3 \mathrm{~mm}$ ) takes 2 minutes with 8 passes and the machining accuracy can remain within $\pm 0.5 \mathrm{~mm}$ (Fig. $9 \mathrm{~b}$ ).

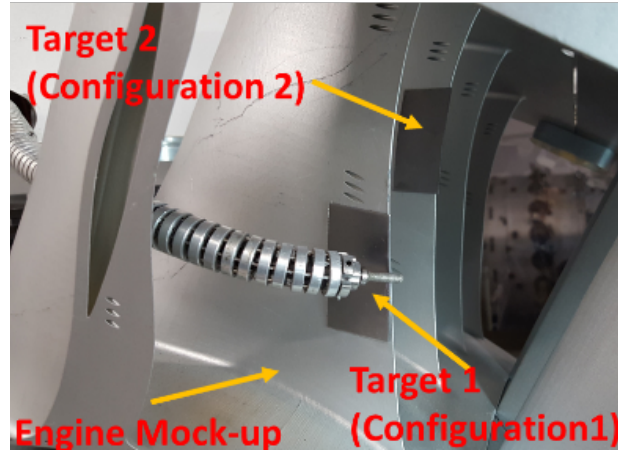

(a)

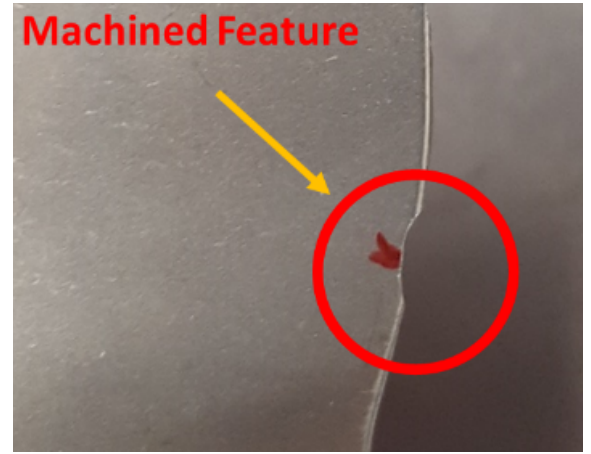

(b)

Fig. 9. Test setup in the engine mock-up (the robot is mounted in the front of the engine mock-up, and navigate to the nozzle guide vanes for repair) (a); one example of the $3 \mathrm{~mm}$ wide machined features (b); 
Table 1. Table of machining results

\begin{tabular}{cccccccc}
\hline \multirow{2}{*}{ Test } & \multicolumn{2}{c}{ Test $(\mathrm{mm})$} & \multicolumn{2}{c}{ Target $(\mathrm{mm})$} & \multicolumn{2}{c}{ Error $(\mathrm{mm})$} & \multirow{2}{*}{ CONFIG } \\
\cline { 2 - 6 } & $\mathrm{P}_{1} \mathrm{P}_{2}$ & $\mathrm{P}_{3} \mathrm{P}_{4}$ & $\mathrm{P}_{1} \mathrm{P}_{2}$ & $\mathrm{P}_{3} \mathrm{P}_{4}$ & $\mathrm{P}_{1} \mathrm{P}_{2}$ & $\mathrm{P}_{3} \mathrm{P}_{4}$ & \\
\hline 1 & 2.82 & 0.88 & 3 & 0.5 & 0.18 & 0.33 & 1 \\
2 & 3.27 & 0.59 & 3 & 0.5 & 0.27 & 0.09 & 2 \\
\hline
\end{tabular}

Based on the in-situ repair standards of aero-engine, the machining accuracy of the continuum robot can sufficiently meet the required quality ( $\max \pm 1 \mathrm{~mm}$ error), bringing a possibility of repairing aero-engines without disassembly, which could offer significant economic advantages for both aero-engine manufacturers and airline operators.

\section{Conclusions}

In this paper, a unique continuum robotic machine tool is presented, which can access and grind aerospace material (e.g. Nickel based alloy) in confined space.

To enable accurate grinding of the stress relief feature, the cable slack was modelled and compensated by an error compensation approach which was developed for reducing the backlash problem caused by the spool mechanism. Then, a new calibration approach is introduced for the in-situ repair operations by the continuum robotic machine tool in cluttered environment. Further, a novel algorithm was developed to generate tool paths for removing the crack (i.e. stress concentration) from the aerospace components (e.g. aero engine compressor), which utilised a new close-form inverse kinematics for 6-DoF continuum robot arm. Finally, by utilising the approaches and algorithm developed in this paper, the machining operations were demonstrated in a confined space (i.e. an aero-engine mock up), and proved the machining accuracy of the system remain within $\pm 0.5 \mathrm{~mm}$.

\section{Acknowledgements}

This work was supported by the FP7 MiRoR project (grant no. 284959 - http://www.miror.eu/) project.

\section{References}

[1] D. J. Smith, "Power-by-the-hour: the role of technology in reshaping business strategy at Rolls-Royce," Technology analysis \& strategic management, vol. 25, no. 8, pp. 987-1007, 2013.

[2] "Richard Wolf," https://www.richard-wolf.com/.

[3] D. Axinte, J. Allen, R. Anderson, I. Dane, L. Uriarte, and A. Olara, "Free-leg Hexapod: A novel approach of using parallel kinematic platforms for developing miniature machine tools for special purpose operations," CIRP Annals-Manufacturing Technology, vol. 60, no. 1, pp. 395-398, 2011.

[4] A. Olarra, D. Axinte, L. Uriarte, and R. Bueno, "Machining with the WalkingHex: A walking parallel kinematic machine tool for in situ operations," CIRP Annals, vol. 66, no. 1, pp. 361-364, 2017.

[5] T. Ota et al., "A highly articulated robotic surgical system for minimally invasive surgery," The Annals of thoracic surgery, vol. 87, no. 4, pp. 1253-1256, 2009.

[6] M. Ho, A. B. McMillan, J. M. Simard, R. Gullapalli, and J. P. Desai, "Toward a meso-scale SMA-actuated MRIcompatible neurosurgical robot," IEEE Transactions on Robotics, vol. 28, no. 1, pp. 213-222, 2012.

[7] W. Xu, T. Liu, and Y. Li, "Kinematics, Dynamics and Control of a Cable-driven Hyper-Redundant Manipulator," IEEE/ASME Transactions on Mechatronics, 2018.

[8] X. Dong, M. Raffles, S. Cobos-Guzman, D. Axinte, and J. Kell, "A novel continuum robot using twin-pivot compliant joints: design, modeling, and validation," Journal of Mechanisms and Robotics, vol. 8, no. 2, p. 021010, 2016.

[9] X. Dong, M. Raffles, S. C. Guzman, D. Axinte, and J. Kell, "Design and analysis of a family of snake arm robots connected by compliant joints," Mechanism and Machine Theory, vol. 77, pp. 73-91, 2014.

[10] K. Xu and N. Simaan, "Analytic formulation for kinematics, statics, and shape restoration of multibackbone continuum robots via elliptic integrals," Journal of Mechanisms and Robotics, vol. 2, no. 1, p. 011006, 2010.

[11] K. Xu, R. E. Goldman, J. Ding, P. K. Allen, D. L. Fowler, and N. Simaan, "System design of an insertable robotic effector platform for single port access (SPA) surgery," in Intelligent Robots and Systems, 2009. IROS 2009. IEEE/RSJ International Conference on, 2009, pp. 5546-5552: IEEE.

[12] B. A. Jones and I. D. Walker, "Kinematics for multisection continuum robots," IEEE Transactions on Robotics, vol. 22, no. 1 , pp. $43-55,2006$. 
[13] S. Cobos-Guzman, D. Palmer, and D. Axinte, "Kinematic model to control the end-effector of a continuum robot for multi-axis processing," Robotica, vol. 35, no. 1, pp. 224-240, 2017. 